\title{
Total Solar Irradiance Observations from the EURECA and ATLAS Experiments
}

\author{
By DOMINIQUE CROMMELYNCK ${ }^{1}$, \\ VICENTE DOMING O , ALAIN FICHOT \\ AND ROBERT LEE III ${ }^{3}$ \\ ${ }^{1}$ Royal Meteorological Institute, Brussels, Belgium \\ ${ }^{2}$ European Space Agency/ESTEC, Noordwijk, The Netherlands \\ ${ }^{3}$ Langley Research Center, Hampton, VA 23681, USA
}

The total solar irradiance was observed by Differential Dual Absolute Radiometers (DIARAD) within the SOVA (SOlar VA riablity) experiment during the EURECA-I mission ( $E U$ ropean $R E$ trievable $C A$ rrier; August 1992 - June 1993) and within the SOLCON (SOL ar CONstant) experiment during the ATLAS-1 and 2 missions (AT mospheric $L$ aboratory for $A$ pplications and $S$ ciences; March 1992 and April 1993). The results from these observations are presented and the metrology aspect addressed through comparison of the simultaneaous measurements with the two DIARADs on EURECA-I and ATLAS-2, respectively.

\section{Introduction}

The SOLCON (SOL ar CONstant) experiment of the ATLAS ( $A T$ mospheric $L$ aboratory for $A$ pplications and $S$ ciences)program is the result of a collaboration between the Royal Meteorological Institute of Belgium (RMIB), the Space Science Department (SSD) of the European Space Agency (ESA), and the Langley Research Center (LRC) of the National Aeronautics and Space Adminiostration (NASA). The SOVA (SOlar VA riablity) experiment on EURECA ( $E U$ ropean $R E$ trievable $C A r r i e r$ ) is lead by RMIB and in cooperation with the Physikalisch Meteorologisches Observatorium Davos (PMOD) and the SSD. Each experiment contains amoung other instruments a Differential Dual Absolute Radiometer (DIARAD) designed by Crommelynck $(1973,1975,1982)$, the results of which are reported here. SOLCON contains also a 2 channel sunphotometer from RMIB and was flown on the ATLAS-1 and 2 missions launched by the Space Shuttles Atlantis and Endeavour on March 24, 1992 and April 7, 1993, respectively. SOVA contains further one 2-channel sunphotometer from RMIB, two PMO6 type absolute radiometers, two 3-channel sunphotometers, one high resolution radiometer and a high precision two axis sun sensor from PMOD. SOVA on EURECA was launched by Atlantis on July 31, 1992 and was brought back to Earth in June 1993 after a flight of more than nine months. The particular interest of this latter mission is that the exeriment is recovered and the performance of the instruments can be checked after the extended exposure to the space environment.

\section{Objectives and instrumentation}

The main scientific objective of the SOVA and SOLCON experiments is the observation of the solar constant and its variation in time. The SOVA experiment observed solar irradiance quasi-continuously for more than 9 months, whereas SOLCON measured during the four and five solar observation periods of ATLAS-1 and 2, respectively. The latter experiment is the continuation of the measurements on Spacelab 1 (Crommelynck \& Domingo 1984). The data of the recent ATLAS missions are mainly aimed at 
comparisons between the different radiometers in space: ACRIM II on UARS (Upper Atmosphere Research Satellite of NASA), solar monitor on ERBS (Earth Radiation Budget Satellites of NASA), HF on NIMBUS 7 (NASA/NOAA), SOVA on EURECA and the other ACRIM on ATLAS. This will improve the metrology of total solar irradiance and aims eventually at the materialization of a World Space Radiometric Reference. Such a reference is needed to support the long-term accurate monitoring of the Sun (Crommelynck 1988).

The SOVA experiment is described in detail by Crommelynck et al. (1993). As the emphasis here is on DIARAD, some details of the data acquisition system and the instrument will be presented. The data acquisition is based on voltage-to-frequency converters which are calibrated against highly accurate reference voltages every 3 minutes. The measuring period extends over $8.2 \mathrm{~s}$ and yields for the measured voltages a resolution of 22 bits. The DIARAD has two cylindrical cavities with a flat bottom which are coated with a diffuse black paint and mounted on a common heat sink with a heat flux sensor in between. The receiver area is circular with a diameter of $10 \mathrm{~mm}$. In contrast to most other solar radiometers, the two detectors are located side by side and both can be used for irradiance measurements which are identified as $-L$ and $-R$ (left and right). The symmetrical arrangement allows for true differential operation which automatically eliminates the effect of changing thermal environment by e.g. changing the temperature of the view-limiting muffler in front of the detectors. In addition, it allows for 16 different radiometric states, used for internal tests and redundancy in operation. During normal mode of operation one detector remains shaded and the other is alternatively irradiated and shaded; Both are heated electrically and the difference in output of the two heat flux sensors is used to drive a servo system which keeps the differential heat fields constant. The shutter is actuated every 99 seconds and the irradiance calculated from a function of the difference between electrical powers in the shaded and irradiated state. Both channels are completely and independently characterized in air and vacuum in order to determine the deviations from ideal behaviour. The uncertainty of the coefficients determined by the characterization provides an evaluation of the absolute accuracy of the detectors which is estimated for DIARAD to be $<0.1 \%$.

\section{Results from the SOVA-DIARAD on EURECA}

On August 10, 1992 the SOVA experiment was switched on and, except for a few days, was pointed at the Sun continuously during nine months up to the time when EURECA was lowered to the rendez-vous orbit for the retrieval. In this orbit SOVA was powered up again and measurements were continued, but with reduced pointing accuracy. SOVADIARAD-L was run all the time and was compared to SOVA-DIARAD-R about every two months for a few orbits. An aging of SOVA-DIARAD-L was observed and was taken into account in the evaluation of the final data which are shown in Figure 1. The results of SOVA-PMO6 (Romero et al. 1994) are very similar and the relative difference at least one order of magnitude smaller than during comparison on the ground. This demonstrates the high quality of data which can be gained from space.

\section{Results from SOLCON-DIARAD on ATLAS 1 and 2}

During the ATLAS 1 and 2 missions both channels of SOLCON-DIARAD were operated and compared in such a way that the exposure of SOLCON-DIARAD-L was always longer than that of SOLCON-DIARAD-R. In the long run, after several ATLAS missions, this will enable us to identify a possible aging due to solar exposure. The comparative 


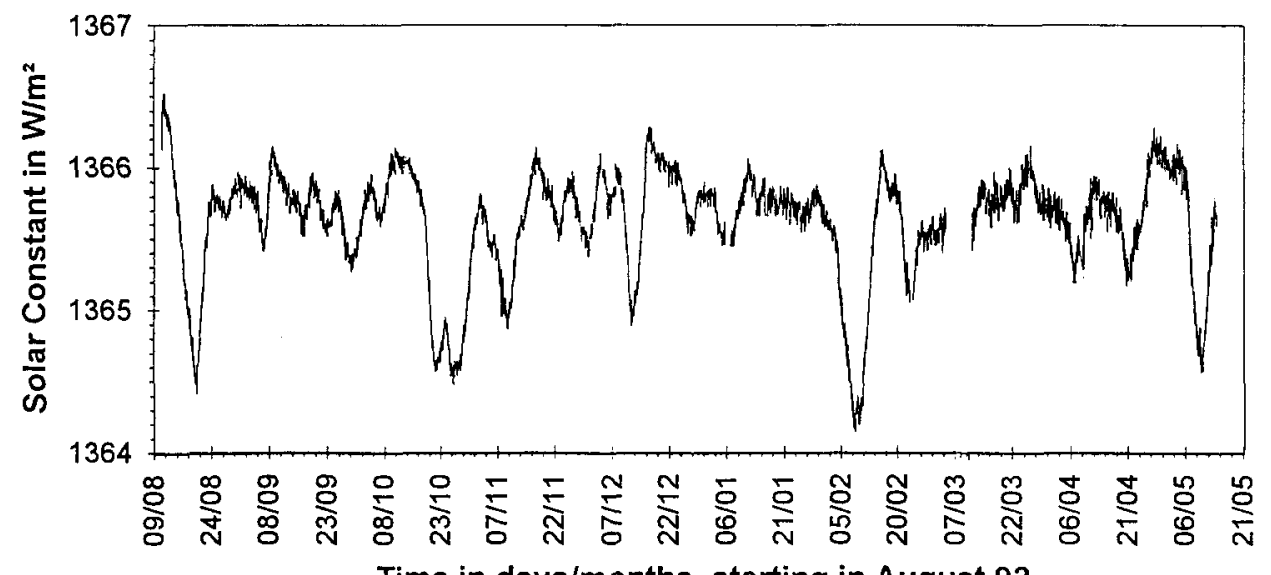

FIGURE 1. Total solar irradiance measured with SOVA-DIARAD-L on EURECA plotted as orbital means. These values shall be multiplied by 1.00091 to reflect the results of the ensemble average of the DIARADs determined by comparisons in space (Table 3 ).

$\begin{array}{lcccc}\text { Observation period } & 1 & 2 & 3 & 4 \\ \text { Day } & 085 & 089 & 091 & 092 \\ \text { Time (UT) } & 17: 00 & 04: 30 & 17: 30 & 20: 30 \\ \text { DIARAD-L } & 1367.65 & 1366.20 & 1366.56 & 1367.15 \\ \text { DIARAD-R } & 1366.92 & 1365.37 & 1365.80 & - \\ \text { Difference } & 0.73 & 0.83 & 0.76 & - \\ \text { Mean } & 1367.29 & 1365.79 & 1366.18 & - \\ \text { 99\% confid. int. } & 0.93 & 1.06 & 0.97 & -\end{array}$

TABLE 1. Results of total solar irradiance determinations reduced to 1 AU obtained by DIARAD during 4 observation periods on the ATLAS 1 mission. All values are in $\mathrm{Wm}^{-2}$.

$\begin{array}{lccccc}\text { Observation period } & 1 & 2 & 3 & 4 & 5 \\ \text { Day } & 099 & 101 & 103 & 105 & 106 \\ \text { Time (UT) } & 07: 30 \text { to } 12: 30 & 15: 45 & 13: 00 & 19: 10 & 09: 45 \\ \text { DIARAD-L } & 1367.15 & 1367.44 & 1367.39 & 1367.31 & 1367.27 \\ \text { DIARAD-R } & 1366.25 & 1366.65 & 1366.61 & 1366.53 & 1366.64 \\ \text { Difference } & 0.9 & 0.79 & 0.78 & 0.78 & 0.63 \\ \text { Mean } & 1366.70 & 1367.04 & 1367.00 & 1366.92 & 1366.96 \\ \text { 99\% confid. int. } & 1.15 & 1.01 & 1.00 & 1.00 & 0.81\end{array}$

TABLE 2. Results of total solar irradiance determinations reduced to $1 \mathrm{AU}$ obtained by DIARAD during 5 observation periods on the ATLAS 2 mission. All values are in $\mathrm{Wm}^{-2}$.

numbers are provided only at the moment that the switching is made between both channels.

The results are presented in Tables 1 and 2 and in Figures 2 and 3 together with the Preliminary Photometric Solar Index (PPSI from Sunspot Bulletin of the Sunspot Index Data Center, Bruxelles) to indicate the level of activity during the observations. Even during the short period of the ATLAS 1 mission one could observe the effect of spots crossing over the solar disk. During the ATLAS 2 mission the Sun was very quiet, not a single sunspot was visible during several days. The differences between the left and right 

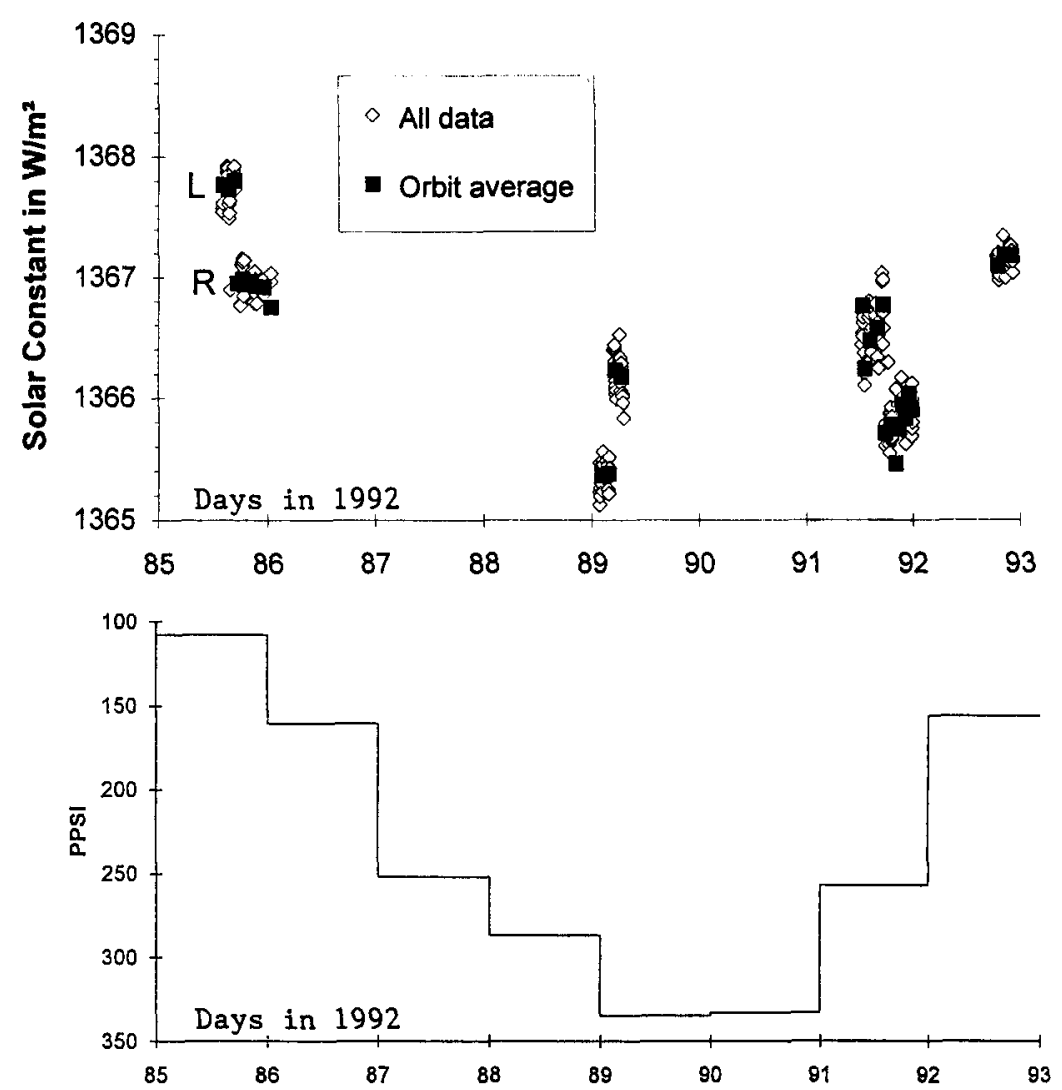

FIGURE 2. Total solar irradiance measured with SOLCON-DIARAD during the ATLAS 1 mission. The PPSI (Provisonal Photometric Sunspot Index, plotted upside down) is shown as indication of the level of activity during the observations.

channels did not show any significantly detectable aging. The ATLAS-1 results could be compared to the measurements obtained with ERBS and the mean solar constant value of SOLCON-DIARAD-L and $\mathrm{R}$ is about $1.5 \mathrm{Wm}^{-2}$ higher than those obtained by ERBS at the same time.

\section{Space absolute radiometric comparison}

During the ATLAS 2 mission, observations of the solar constant was obtained quasisimultaneously with DIARAD in SOLCON and SOVA. Fortunately the Sun was very quiet during these observation periods so that true simultaneity was not so important. The four values obtained during the different solar performances of the ATLAS-2 mission. can thus be used each time to determine an average with its confidence interval of the population mean (at e.g. 99\% confidence level). The aging of SOVA-DIARAD-L was corrected before the determination of the mean. The results presented in Table 3 provide in fact an objective state-of-the-art status of the accuracy that is obtained with DIARAD. If we consider only SOLCON-DIARAD-R, SOVA-DIARAD-R and SOLCON-DIARAD-L, the value of their mean would have a confidence interval of $0.7 \mathrm{Wm}^{-2}$. We believe that the value of the SOVA-DIARAD-L sensitive area is probably known with less accuracy than the three other channels. However SOVA-DIARAD-L can not be eliminated arbi- 


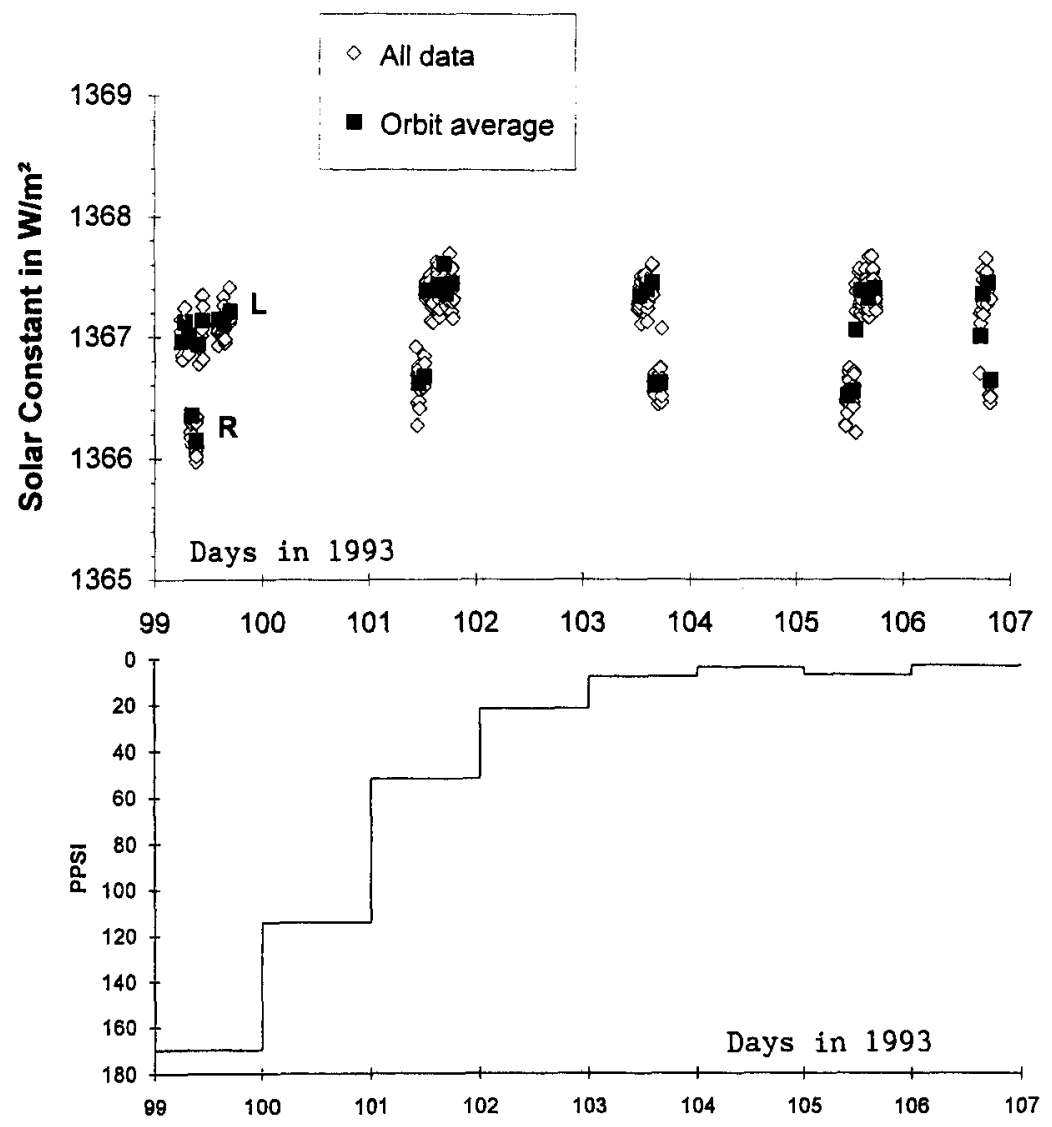

FIGURE 3. Total solar irradiance measured with SOLCON-DIARAD during the ATLAS 2 mission. The PPSI (Provisonal Photometric Sunspot Index, plotted upside down) is shown as indication of the level of activity during the observations.

trarily from the population of instruments. Note that the $99.9 \%$ confidence interval of about $0.15 \mathrm{Wm}^{-2}$ on the measurements with one channel is partly due to instrumental noise and partly to solar noise. The relative contributions is under investigation.

Table 3 shows also that radiometric comparisons in space can be done with a much better precision than on the ground where the atmospheric turbidity and transmittance variability are the limiting effects. The space calibration of a stable high precision relative radiometer with an accurate and well defined absolute radiometer is limited only by the state-of-the-art absolute accuracy and the precision of both instruments. The results were obtained on April 11,13 and 15,1993 with very stable mean solar constant values respectively of $1366.49,1366.45$ and $1366.44 \mathrm{Wm}^{-2}$. The evaluated absolute error or uncertainty of these values is consistent with the measured differences at $99 \%$ confidence level.

It must be realised that the only way to improve further the accuracy and thus the knowledge of the real solar constant is to compare our values to other simultaneous observations also made with highly accurate absolute (in a strict sense) radiometers. It is then possible to calculate a new mean that would improve the actual confidence interval. However, if another set of measurements exists with a similar or better confidence 


Date 1993
Time (UT)
SOVA-DIARAD-L
$99.9 \%$ conf. int.
SOLCON-DIARAD-R
99.9\% conf. int.
SOVA-DIARAD-R
99.9\% conf. int.
SOLCON-DIARAD-L
99.9\% conf. int.
Mean
$99 \%$ conf. int.

$\begin{array}{cccc}9 \text { April } & \text { 11 April } & \text { 13 April } & \text { 15 April } \\ 08: 00-17: 30 & 09: 00-18: 00 & 11: 00-19: 00 & 07: 30-20: 00 \\ 1364.92 & 1365.23 & 1365.21 & 1365.24 \\ 0.14 & 0.13 & 0.17 & 0.12 \\ 1366.25 & 1366.65 & 1366.61 & 1366.53 \\ 0.16 & 0.15 & 0.12 & 0.10 \\ 1366.37 & 1366.64 & 1366.58 & 1366.68 \\ 0.14 & 0.12 & 0.16 & 0.40 \\ 1367.15 & 1367.44 & 1367.39 & 1367.31 \\ 0.12 & 0.12 & 0.14 & 0.16 \\ 1366.17 & 1366.49 & 1366.45 & 1366.44 \\ 1.42 & 1.47 & 1.46 & 1.43\end{array}$

TABLE 3. Total solar irradiance observations with SOLCON-DIARAD on ATLAS 2 and SOVA-DIARAD on EURECA I. All values are in $\mathrm{Wm}^{-2}$. The aging of SOVA-DIARAD-L has been removed.

interval but with a mean outside our confidence interval this would be the indication of a metrological problem and/or of a wrong estimate of the uncertainty.

It is important and necessary that each design of an absolute radiometer has its uncertainty determinded by experimental characterization. A set of such instruments can then be compared by measuring the same stable source and the results be used to verify the uncertainty which can be achieved with this design (for DIARAD see Table 3 ).

\section{Conclusions}

The concept of DIARAD in SOLCON and SOVA has been proven to be efficient and has been operated succesfully on the ATLAS 1 and 2 missions and on EURECA. For the first time four detectors characterised independently have measured quasi-simultaneous the solar irradiance from space each with a precision of much better than $0.01 \%$ and a $99 \%$ confidence interval uncertainty of the mean of $\pm 0.1 \%$ at a $99 \%$ confidence level.

Based on preliminary results ERBS, UARS/ACRIM II and PMO6-SOVA (referenced to the World Radiometric Reference on ground) are close to the mean of Table 3.

Acknowledgments. The RMIB was funded by the Fonds de la Recherche Fondamentale Collective d'Initiative Ministérielle. The quality of the engineering support of A. Chevalier, J. Donaldson, B. Penelle, U. Telljohann and their colleagues, the team members of the three collaborating institutions as well as the supporting teams of the space agencies resulted in an efficient and successful group achievement. C. Fröhlich is acknowledged for discussing and editing our manuscript.

\section{REFERENCES}

Crommelynck, D. 1973 Théorie instrumentale en radiométrie absolue. I.R.M. Publication série $A \mathbf{8 1}, 1-50$.

CRommelynck, D. 1977 Calibration of radiation instruments for measurement of the radiant flux of an arbitrary Source. In Symposium on Optical Sensing and Probing of the Atmosphere (IS 23) - Assemblée Générale de l'UGGI, Grenoble (F), 1975. Applied Optics 16, 302-305. 
CRommelynck, D. 1982 Fundamentals of absolute pyrheliometry and objective characterization. Earth Radiation Science Seminars, NASA Conference Publication 2239, 53-88.

Crommelynck, D. 1985 The IRMB/SSD Absolute Radiometric Base, Objectives and Developments, Advances in Absolute Radiometry for Atmospheric and Environmental Research, Inc., Cambridge, Massachussetts, pp. 12-15.

Crommelynck, D. 1988 Factors limiting the accuracy of absolute radiometry. In New developments and applications in optical radiometry Institute of Physics Conference series number 92, NPL, London, 19-24.

Crommelynck, D. \& Domingo, V. 1984 Solar irradiance observations. Sciences 225, 180-181.

Crommelynck, D., Domingo, V., Fichot, A., Fröhlich, C., Penelle, B., Romero J. \& WEHRLI, CH. 1993 Preliminary Results from the SOVA Experiment on Board the European Retrievable Carrier (EURECA). Metrologia 30, 372-379.

Romero, J., WeHrLI, C. \& Fröhlich, C. 1994 Solar total irradiance variability from SOVA 2 on board EURECA, Solar Phys. 152, in press. 